\title{
Analysis on the Performance of the Balb/c 3T3 Two-stage Assay in a Miniature Culture Area and in a Perfusion Culture Model
}

\author{
Vania H. M. TEOFILO ${ }^{1}$, Jin-Tao LI, a, ${ }^{1}$, Xin-Hui LIU ${ }^{1, b}$, \\ Ru-Gang ZHONG ${ }^{1}$, Si YE ${ }^{1}$, Zheng-Bo ZHAO ${ }^{1}$, Yi ZENG ${ }^{2}$ \\ ${ }^{1}$ Beijing Key Lab. of Environmental \& Viral Oncology, College of Life Science \& \\ Bioengineering, Beijing Univ. of Technology, Beijing, 100124, China \\ ${ }^{2}$ State Key Laboratory for Infectious Disease Prevention and Control, National \\ Institute for Viral Disease Control and Prevention, Chinese Center for Disease \\ Control and Prevention, Beijing,100052, China \\ aemail: ljt2000593@163.com, bemail: liuxinhui@bjut.edu.cn
}

Keywords: CTAs, Balb/c 3T3, Perfusion cell culture.

\begin{abstract}
The Balb/c 3T3 cell transformation assay (CTA) is presently undergoing extensive pre-validation testing but its potential for routine chemical screening remains to be fully exploited due it being more costly and more time consuming than other CTAs. To downscale the assay 24-well plates were used to score foci and then compared to the standard T25 culture bottles. Furthermore, it was also attempted an adaptation to a perfusion cell culture model in order to achieve a closer equivalent to the in vivo carcinogenic processes. The miniaturized approach was successfully correlated to the standard technique. A reproduction in smaller culture plates can provide an alternative for a faster and more practicable handling by smaller laboratories. The assay failed to produce positive results in the fluidic model and further testing is necessary to determine the role medium flow in the in vitro carcinogenesis process.
\end{abstract}

\section{Introduction}

In vitro cell transformation assays (CTAs) for the screening of chemical carcinogenic potential are becoming increasingly relevant as they aim to reduce or substitute rodent life-time bioassays that have been shown to be costly, time-consuming and, furthermore, true predictivity of animal carcinogenicity data to humans has been questioned [1-7].

One of the CTAs undergoing pre-validation testing by the European Center for Validation of Alternative Methods (ECVAM) is the Balb/c 3T3 assay which analyzes chemical induced morphological transformed foci [8].

This assay was developed by Kakunaga in 1973 using a A31-714 clone derived from the mouse Balb/c 3T3 A31 embryonic cell line [9] which demonstrated a high degree of contact inhibition, growing in a single monolayer and, never piling up, unless when treated with a carcinogen at the beginning cultivation [10].

The standard assay involves the seeding of $60 \mathrm{~mm}$ culture dishes or $25 \mathrm{~cm}^{2}$ culture flasks with approximately $1 \times 10^{4}$ cells that are treated 24 hours after seeding with a test chemical for 72 hours after which cells are cultured for an additional four to six weeks [11]. A Colony Forming Efficiency test (CFE) may be performed followed by the Morphological Transformation Assay (MTA), in which seven doses may be tested in addition to a control test and negative control test allowing for a better standardization and inter-laboratories reproducibility $[12,13]$. 
The assay has undergone some modifications, such as the use of DMEM/F12 medium supplemented with 2\% Bovine Serum [14] and the implementation of a two stage transformation assay for the screening of initiating and promoting activities of chemicals. At the beginning of cultivation cells are treated with a sub-threshold dose of a carcinogen, a tumor initiator, and, afterwards, with a tumor promoter thus mimicking in vivo two-stage carcinogenesis process $[15,16]$. Both modifications were found to be relevant, improving the scoring of foci for different tested substances [17].

Even with these modifications and the substantial amount of data on reproducibility, sensitivity and specificity collected, the Balb/c 3T3 CTA remains to be fully exploited since it's technically more difficult, expensive and time consuming than other in vitro genotoxicity tests [13].

Recently a study performed on the miniaturization another widely used CTA, the Syrian Hamster Embryo (SHE) cells assay, demonstrated not only that the test was reproducible in a smaller scale but that is allowed for faster performance while utilizing a smaller number of cells [18].

A miniature approach can also facilitate the adaptation of the assay to a perfusion cell culture, which may, in turn, provide a closer approximation to an in vivo environment furthering our understanding of the carcinogenesis process.

Perfusion cell culture can provide a good alternative to static cell culture by incorporating a system of nutrient supply and waste removal keeping a more stable environment [19].The medium flow can mimic vascular flow responsible for distribution of soluble factors [20]. However, fluidic systems also present their own set of obstacles: cell seeding might be compromised by the shear stress of fluidic flow and, the constant flow can remove essential soluble biomolecular factors involved in cell-cell dynamic [19-22].

In this study we aimed to downscale the standard Balb/c 3T3 two-stage transformation assay. We also attempted to implement the assay in a perfusion cell culture technique to access the possible effects of a continuous fluidic flow on the cell transformation process.

\section{Materials and Methods}

\section{Cell Line and Culture Maintenance}

Balb/c 3T3 cells were provided by the State Key Laboratory for Infectious Disease Prevention and Control, National Institute for Viral Disease Control and Prevention, Chinese Center for Disease Control and Prevention. They were originated from ATCC CCL-163 Balb/c mouse whole embryo cultures. These cells possess the ability to divide indefinitely and are highly sensitive to post-confluence inhibition of cell division.

Cells were kept in T25 culture flasks at $37^{\circ} \mathrm{C}, 5 \% \mathrm{CO}^{2}$ and were passaged every three days before reaching $70 \%$ confluence.

DMEM/10F medium was used in routine cell maintenance and initial stage of the two-stage transformation protocol. Medium was kept at $4^{\circ}$ and contained Dulbecco's Modified Eagle's Medium (Hyclone), 10\% (v/v) Fetal Bovine Serum (FBS, Hyclone) and $1 \%$ (v/v) Penicillin/Streptomycin solution (10,000units/ml, Hyclone).

DMEMF12/2F medium was used in later stage of the two-stage transformation protocol and contained DMEM/F12 (1:1) (Hyclone) complemented with 2\% FBS, 1\% Penicillin/Streptomycin solution and $0.2 \% \quad(\mathrm{v} / \mathrm{v})$ ITES (containing $1.0 \mathrm{mg} / \mathrm{ml}$ recombinant human insulin, $0.55 \mathrm{mg} / \mathrm{ml}$ human transferrin, $0.5 \mu \mathrm{g} / \mathrm{ml}$ sodium selenite, 
$0.2 \mathrm{mg} / \mathrm{ml}$ ethanolamine, from Sigma-Aldrich).

\section{Two Stage Transformation Assay}

In the traditional assay $1 \times 10^{4}$ cells are seeded into T25 $\left(25 \mathrm{~cm}^{2}\right.$ culture area) cell cultures flasks or $60 \mathrm{~mm}$ culture plates (aprox. $400 \mathrm{cells} / \mathrm{cm}^{2}$ ). Since Corning 24 -well culture plates have an actual growth area of $2 \mathrm{~cm}^{2}$, the initial seeding concentration was calculated to be 800 cells/well, however this concentration failed to achieve confluence before treatment with tumor promoter and the seeding concentration was rounded up to 1000 cells/well $\left(1 \times 10^{3}\right)$.

\section{Time Line}

Day 0: Cells were seeded into T25 cultures flasks (Corning) and 24-well plate and kept overnight on DMEM/10F medium at $37^{\circ} \mathrm{C}, 5 \% \mathrm{CO}$. Day 1: 24 hours after seeding, the culture medium was replaced for DMEMF12/2F medium containing $0.2 \mu \mathrm{g} / \mathrm{ml} 3$-methylcholanthrene (MCA). Day 4: 72 hours after exposure the treatment medium was replaced with DMEMF12/2F medium. On Day 7 the medium was replaced with DMEMF12/2F containing $0.1 \mu \mathrm{g} / \mathrm{ml}$ of tumor promoter 12-Otetradecanoylphorbol (TPA). Cells were treated with TPA for a week with one replacement of fresh, TPA added DMEMF12/2F medium either on Day 10 or 11 . A week after treatment (Day 14) medium was once again replaced with DMEMF12/2F (without TPA) which was replaced every 3 days until day 26. On Day 31 (5 days after last medium change) cells were stained in Giemsa solution, air dried and number of foci was recorded. The assay was repeated twice (groups A and B), each time five T25 culture flasks + control and three 24 -well plates (60 treatment wells and 12 control) were used.

\section{Culture in TissueFlex ${ }^{\circledR}$ Micro Bioreactors (TFBR) System}

The TFBR consists of a four well unit (TG 4-well) formed from a gas permeable biocompatible polymer. Each well has an inlet and outlet port allowing for medium intake and waste removal respectively. Each inlet has a syringe connector tube that allows individual perfusion. Perfusion rates can be precisely controlled and adapted to the two-stage assay medium changes. The TFBR is kept in a specially designed bench top incubator which incorporates a PID controller for temperature selection and $\mathrm{CO}^{2}$ levels are maintained by tubing connecting its lid to a $\mathrm{CO}^{2}$ a control unit. The incubator can hold two TFBR at a time and four repetitions were performed (32 wells: 24 testing +8 controls).

Since the Balb/c 3T3 two-stage transformation assay relies on disruption of the post-confluence cell division inhibition, cells were kept in a monolayer for a 2D perfusion cultures. Assuming that the wells of the TG 4-well bioreactor are comparable in size with the wells of a 24-well plate, cells were seeded at concentrations of 1000 cells/well. After seeding cells were kept for 24 hours in regular $\mathrm{CO}^{2}$ incubator. On Day 1 medium was removed and cells were transferred to the bench top incubator. Syringes were filled with $3 \mathrm{ml}$ of MCA added DMEM/10F medium, $1 \mathrm{ml}$ was injected in each well and the remaining $2 \mathrm{ml}$ was injected at a rate of $0.46 \mu 1 /$ minute for the next three days. All medium changes were done in this manner and corresponded to the same time line described above.

\footnotetext{
*TissueFlex® Micro Bioreactors (TG-4 wells) and supportive machinery (TissueFlex® Incubator, TissueFlex® Syringe Pump) were purchased from Zyoxel Ltd.
} 


\section{Foci Scoring}

Foci containing more than 50 cells or measuring more than $2 \mathrm{~mm}$ in diameter were scored. In addition, the photo catalogue for the classification of foci in the BALB/c 3 T3 cell transformation assay by Sasaki et al. 2012a [23] and Urani et al. 2013 [7] were used as a visual aid for the identification of Type III foci.

\section{Results}

\section{Foci Scoring in 24-well Plates and T25 Culture Flask}

Number of foci in 24 -well plates was scored per $24 \mathrm{~cm}^{2}$ area (12 wells) designated as W (Table 1) and only Type III foci were scored (Fig. 1). Number of foci per W area was relatively low ( $\leq 10$ /scoring area) with a total of 81 foci for the ten tested $\mathrm{W}$ areas.

Table 1. Foci distribution in 24-well plates

\begin{tabular}{|c|c|c|c|c|c|c|c|c|c|c|c|c|c|}
\hline \multicolumn{7}{|c|}{ Group A } & \multicolumn{7}{|c|}{ Group B } \\
\hline & $\mathbf{1}$ & 2 & 3 & 4 & 5 & 6 & & $\mathbf{1}$ & 2 & 3 & 4 & 5 & 6 \\
\hline A & o & 1 & 2 & o & 2 & 1 & $\mathbf{A}$ & 1 & 1 & O & 1 & O & 1 \\
\hline $\mathbf{B}$ & 1 & 0 & 1 & 1 & 0 & 1 & B & $\mathrm{o}$ & 0 & 0 & 2 & 1 & 1 \\
\hline C & 1 & o & o & 1 & 1 & o & C & 1 & 1 & 1 & $\mathrm{O}$ & 1 & $\mathrm{O}$ \\
\hline \multirow[t]{3}{*}{ D } & o & 1 & 1 & 0 & 1 & 1 & D & o & 0 & 1 & $\mathrm{O}$ & 1 & $\mathrm{O}$ \\
\hline & \multicolumn{3}{|c|}{ W1 } & \multicolumn{3}{|c|}{ W2 } & & \multicolumn{2}{|c|}{ W6 } & & \multicolumn{2}{|c|}{ W7 } & \\
\hline & 1 & 2 & 3 & 4 & 5 & 6 & & 1 & 2 & 3 & 4 & 5 & 6 \\
\hline A & o & 1 & 2 & o & 1 & 1 & $\mathbf{A}$ & O & 1 & $\mathrm{O}$ & 1 & $\mathrm{O}$ & 1 \\
\hline $\mathbf{B}$ & 1 & 1 & 0 & 1 & 0 & 1 & $\mathbf{B}$ & 0 & 1 & 2 & 0 & 2 & 0 \\
\hline C & o & 1 & o & 1 & 1 & 1 & C & o & 2 & 1 & 2 & o & 1 \\
\hline \multirow[t]{3}{*}{ D } & 1 & 0 & o & 1 & 2 & o & D & 1 & o & 0 & $\mathrm{O}$ & 1 & 1 \\
\hline & \multicolumn{3}{|c|}{ W3 } & \multicolumn{3}{|c|}{ W4 } & & \multicolumn{2}{|c|}{ W8 } & & \multicolumn{2}{|c|}{ W9 } & \\
\hline & 1 & 2 & 3 & 4 & 5 & 6 & & 1 & 2 & 3 & 4 & 5 & 6 \\
\hline $\mathbf{A}$ & 2 & 1 & 1 & 0 & o & o & $\mathbf{A}$ & o & 0 & 1 & O & $\mathrm{O}$ & 0 \\
\hline B & 0 & 2 & 1 & 0 & 0 & 0 & $\mathbf{B}$ & 1 & 0 & 1 & $\mathrm{O}$ & 0 & 0 \\
\hline C & 1 & 0 & 0 & 0 & 0 & 0 & C & 1 & 0 & 1 & 0 & 0 & 0 \\
\hline \multirow[t]{2}{*}{ D } & 1 & 0 & 0 & 0 & 0 & 0 & D & 1 & 0 & 1 & 0 & 0 & 0 \\
\hline & \multicolumn{3}{|c|}{ W5 } & \multicolumn{3}{|c|}{ Control a } & & \multicolumn{3}{|c|}{ W10 } & \multicolumn{3}{|c|}{ Control b } \\
\hline
\end{tabular}

The number of foci scored did not differ significantly between T25 flask and 24-well plates (Fig. 2) Overall Type III foci number was low in both culture vessels, with foci numbers remaining $\leq 10$ per flask and per $24 \mathrm{~cm}^{2}(\mathrm{~W})$. In total 71 foci were scored in the T25 culture flask trial.
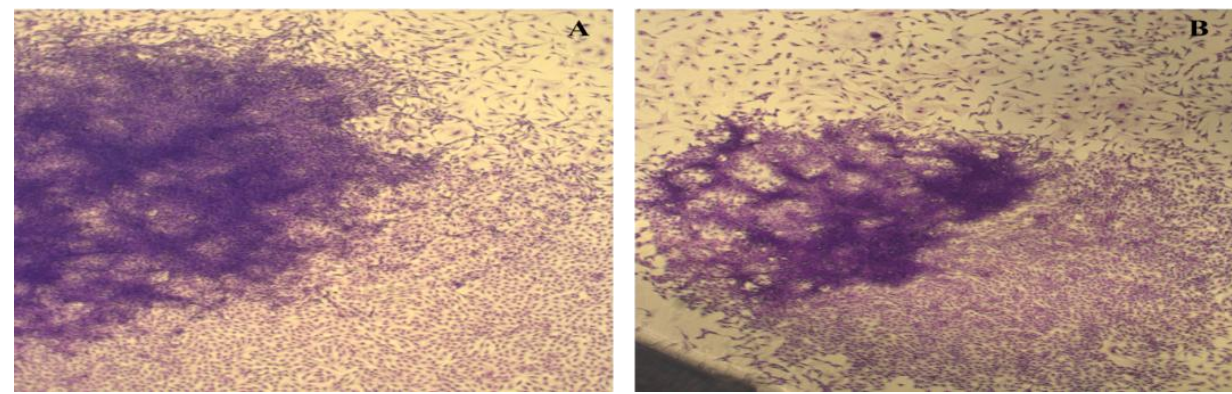

Figure 1. Transformed foci in: A) T25 culture flask and B) 24 well plate. 

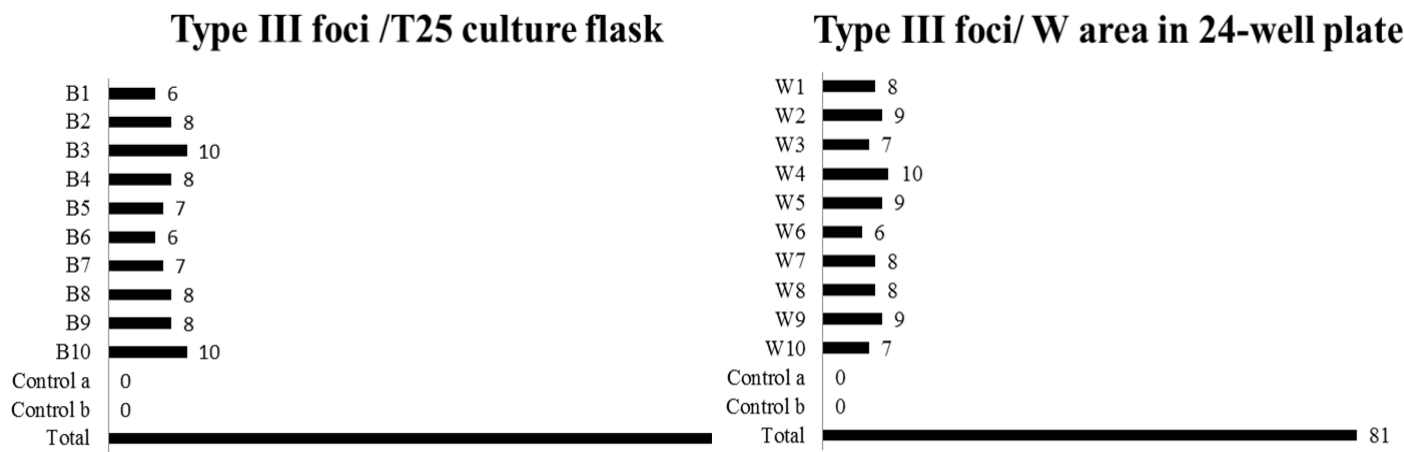

Figure 2. Type III foci scored per T25 culture flask and $24 \mathrm{~cm}^{2}$ of 24 -well plate (W).

The average number of transformed foci per T25 culture flask and $\mathrm{W}$ area were 7.8 and 8.1 respectively. No significant difference was found between the means of the two groups when compared by T-test $[\mathrm{p} \geq 0.05]$.

Table 2.Comparison between the means of foci number/T25 flask vs W (12wells)

\begin{tabular}{lccl}
\hline & T25 bottles & $\begin{array}{c}\text { Well plates } \\
(\mathbf{W})\end{array}$ & \\
\hline Mean & 7.8 & 8.1 & T-Test [p-value] \\
Variance & 1.955 & 1.433 & $\mathbf{P} \geqslant \mathbf{0 . 0 5}$ \\
SD & 1.398 & 1.197 & \\
\hline
\end{tabular}

\section{Adaptation of Balb/c 3T3 Assay to Perfusion Bioreactor}

On the initial phase of the assay cells seeded into the TFBR presented a more even distribution of cells and rapid cell growth compared to the static wells where, cells tend to first grow around the periphery slowly moving to the center. In addition, confluence was achieved faster, however, the experiment failed to produce a significant number of Type III foci. A second trial was attempted with a seeding concentration of $2 \times 10^{3}$ cells/well. It also failed to produce foci that could be considered be scored. In both trials, small transformed foci were observed but failed to achieve the sizes of foci observed in the standard assay (Fig. 3).

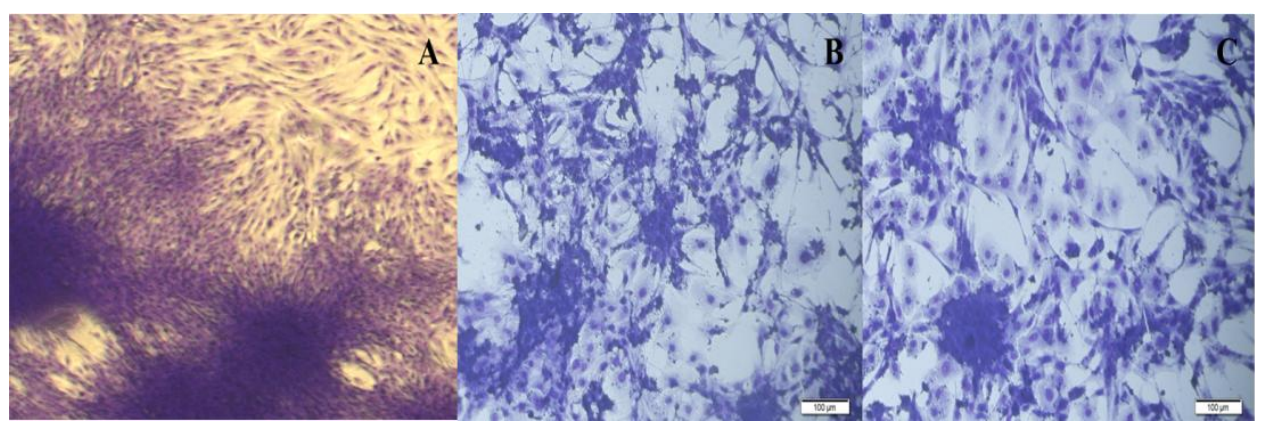

Figure 3. A) Border section of Type III foci in T25 flask presenting deep basophilic staining in contrast with the small foci on TFBR with B) $1 \times 10^{3}$ seeding concertation and C) $2 \times 10^{3}$ seeding concertation. 


\section{Discussion}

\section{Miniature Culture Area VS Standard Culture Area}

To predict the carcinogenic potential of chemical compounds, life-time rodent bioassays have, traditionally, been the standard method [1-3]. Such assays are time consuming and expensive, usually taking up to three years and involving the use of 600-800 animals per chemical and the histo-pathological examination of more than 40 tissues $[4,5]$. In vitro transformation assays are faster, more cost efficient and display a multistep process that closely models the multistage process of carcinogenesis $[1,8]$.

They have not yet become the standard method in screening chemicals partly because they are still under validation analyses, but also because some are not simple for routine tests [24].

The main goal of this study was to attempt a downscaling the Balb/c 3T3 two-stage transformation assay in order to facilitate smaller laboratories or laboratories where a vast number of chemical are screened to reproduce the assay in a more efficient manner.

For a better correlation to the $25 \mathrm{~cm}^{2}$ culture area of the T25 culture flask the foci on the 24-well plate were scored per 12 wells for an approximate culture area of $24 \mathrm{~cm}^{2}$ (presented as $\mathrm{W}$ area in this study).

We observed that the total number and means of foci did not differ much between the T25 culture flask and W area (78 and $81 ; 7.8$ and 8.1 respectively). In both T25 flasks and 24-well plates presented a low number of foci per scoring area $(\leq 10)$ suggesting that this may be due to the cell batch. The total number of foci was slightly higher in the scoring area of the 24 well plates; this might be due to a higher concentration of cells per $\mathrm{cm}^{2}$. Conversely, a trial experiment with a seeding concentration of $2 \times 10^{3}$ cells/well only presented Type I and II foci (data not shown).

Our results suggest that the two-stage Balb/c 3T3 assay can be reproducible in smaller culture areas which could facilitate the handling and repetition of the assay. Nevertheless, more studies, further repetition and testing are necessary to establish if these results can be reproduced in different laboratories and in order to collect data for a more robust statistical analysis.

Additionally, since in this study, only the basic two-stage compounds where tested ( MCA \& TPA), it would be necessary to conduct experiments with different dose levels of chemicals to confirm if miniaturization will allow for a concentration based analysis. It is also important to mention that, many of the recent validation studies of the Balb/c 3T3 transformation assay have been focused on the one stage assay, with the establishment of improved protocols and new statistical approaches [12, 13, and 25]. It would be therefore, crucial to focus on a miniaturization approach on the one stage assay as well.

\section{Balb/c 3T3 Assay in TFBR Perfusion Culture}

One of the advantages of miniaturization approach is the possibility was to adapt the protocol to other cell culture techniques such as the perfusion model. This technique can provide a carcinogenicity onset model closer to the in vivo vascular flow environment.

On the initial phase of the perfusion bioreactor trial cells presented a more even distribution and achieved confluence at a faster rate but failed to produce sizeable transformed foci with characteristics that would allow scoring. This may indicate that the constant removal of soluble factors interferes with the expansion of transformed 
cells. Recently, studies on tumor microenvironment have suggested that, cancer proliferation does not only rely on somatic mutation, but on soluble factors that act in intracellular mitogenic pathways and can also be signaled to neighboring cells.

In a 2011 review study, Hanahan \& Weinberg remarked that the growth factor signals controlling cell number and position within tissues are thought to be transmitted in a temporally and spatially regulated fashion from one cell to its neighbors in a process that is difficult to analyze experimentally [26]. Other studies have discussed the difficulties of incorporating tumor micro environment factors in standard carcinogenic studies [27, 28]. These studies point to the complexity of modeling screening test that can truly mimic human tissue physiology. The CTAs can mimic the multistage carcinogenesis process but, many chemicals which test positive in the in vitro assays fail to induce tumors in the animal model. It is possible that more detailed analysis on molecular soluble factors implicated in the carcinogenic processes using a perfusion model may prove useful for the understanding of such disparity and a may provide better insight on the in vivo carcinogenic process.

\section{Conclusion}

This miniaturization attempt showed that it is possible to downscale the two stage protocol of the Balb/c 3T3 assay. To continue with a miniaturization approach it will be necessary, in the future to analyze if smaller vessels can provide enough data for dose response curves and if it can be adapted to the standard one stage assay. However considering the long term applications of the assay and, its advantage in foci scoring and no need of irradiation, compared to the SHE assay [13], a downscale approach can aid in the development and increasing of practicality and reproducibility of the assay.

Unfortunately, the perfusion cell culture model, for the conditions that we test seems to be impracticable for the scoring of transformed foci. Nevertheless, the perfusion model may provide an insight ion the role of tumor microenvironment soluble factors in cancer proliferation. Such studies could help develop screening test that better reflect the in vivo cell and tissue physiology without the ethical and cost burdens presented by animal testing.

\section{Acknowledgments}

This study was supported by the following grants:

Grant sponsor: State 863 projects, Grant number: 2012AA02A404.

Grant sponsor: Scientific Research Program of Beijing Municipal commission of Education. Grant number: PXM2014_014204_07_000046.

Grant sponsor: Beijing University of Technology Foundation (015000514314004).

\section{References}

[1] R. Combes, M. Balls, R. Curren, M. Fishbach, N. Fusering, D. Kirkland, C. Lasne, J. Landolph, R. LeBoeuf, H. Marquard, J. McCormick, L. Müller, E. Rivedal, E. Sabbioni, N. Tanaka, P. Vasseur, H. Yamasaki, Cell transformation assays as predictors of human carcinogenicity, ATLA-Altern. Lab. Anim. 27 (1999) 745-767.

[2] Information on http://www.oecd.org/env/testguidelines [Detailed review on cell transformation assays for detection of chemical carcinogens. OECD Environment, Health and Safety Publications (2007), Series on Testing and Assessment n. 31]. 
[3] Information on http://www.oecd-ilibrary.org/environment/test-no-451carcinogenicity-studies_9789264071186-en [Test Guideline 451 - Carcinogenicity studies. OECD Guidelines for the Testing of Chemicals, (2009) OECD, Paris].

[4] P. Vanparys, R. Corvi, M. Aardema, L. Gribaldo, M. Hayashi, S. Hoffmann L. Schechtman, ECVAM prevalidation of three cell transformation assays. Altex 28 (2011) 56-59.

[5] S. Creton, M.J. Aardema, P.L. Carmichael, J.S. Harvey, F.L. Martin, R.F. Newbold, M.R. O'Donovan, K. Pant, A. Poth, A. Sakai, K. Sasaki, A.D. Scott, L.M. Schechtman, R.R. Shen, N. Tanaka, H. Yasaei, Cell transformation assays for prediction of carcinogenic potential: state of the art and future research needs. Mutagenesis 27(1) (2012) 93-101.

[6] J.M. Ward, The two-year rodent carcinogenesis bioassay - Will it survive? J. Toxicol. Pathol. 20 (2007) 13-19.

[7] C. Urani, R. Corvi, G. Callegaro, F.M. Stefanini, Objective scoring of transformed foci in BALB/c 3T3 cell transformation assay by statistical image descriptors. Toxicol.in Vitro 27 (2013) 1905-12.

[8] R. Corvi, M. Aardema, L. Gribaldoc, M. Hayashi, S. Hoffmanne, L. Schechtman

P. Vanparys, ECVAM prevalidation study on in vitro cell transformation assays: General outline and conclusions of the study, Mutat. Res. 744 (2012) 12-19.

[9] T. Kakunaga, A quantitative system for assay of malignant transformation by chemical carcinogens using a clone derived from BALB/3T3, Int. J. Cancer 12 (1973) 463-473.

[10] A. Sakai, Balb/c 3T3 cell transformation assays for the assessment of chemical carcinogenicity, AATEX 14(Special Issue) (2008) 367-373.

[11] E.J. Matthews, Transformation of BALB/c-3T3 cells: I. Investigation of experimental parameters that influence detection of spontaneous transformation, Environ. Health.Perspect. 101(2) (1993) 277-291.

[12] K. Sasaki, S. Bohnenberger, K. Hayashi, T. Kunkelmann, D. Muramatsu, P. Phrakonkham, A. Poth, A. Sakai, S. Salovaara, N. Tanaka, B.C. Thomas, M. Umeda, Recommended protocol for the BALB/c 3 T3 cell transformation assay, Mutat. Res. 744 (2012b) 30-35.

[13] N. Tanaka, S. Bohenenberger, T. Kunkelmann, B. Munaro, J. Ponti, A. Poth, E. Sabbioni, A. Sakai, S. Salovaara, K. Sasaki, B.C. Thomas, M. Umeda, Prevalidation study of the Balb/c 3T3 cell transformation assay for assessment of carcinogenic potential of chemicals, Mutat. Res. 744 (2012) 20-29.

[14] K. Hayashi, K. Sasaki, S. Asada, T. Tsuchiya, M. Hayashi, I. Yoshimura, N. Tanaka, M. Umeda, Technical modification of the Balb/c 3T3 cell transformation assay: the use of serum-reduced medium to optimize the practicability of the protocol, ATLA-Altern. Lab. Anim. 36(6) (2008) 653-65.

[15] K. Sasaki, K. Chida, H. Hashiba, N. Kamata, E. Abe, T. Suda, T. Kuroki, Enhancement by $1 \alpha, 25$-dihydroxyvitamin D3 of chemically induced transformation of BALB 3T3 cells without induction of ornithine decarboxylase or activation of protein kinase C, Cancer Res. 46 (1986) 604-610. 
[16] A. Sakai, H. Fujiki, Promotion of BALB/3T3 cell transformation by the okadaic acid class of tumor promoters, okadaic acid and dinophysistoxin-1, J. Cancer Res, 82 (1991) 518-523.

[17] T. Tsuchiya, M. Umeda, N. Tanaka, A. Sakai, H. Nishiyama, I. Yoshimura, S. Ajimi, S. Asada, M. Asakura, H. Baba, Y. Dewa, Y. Ebe, Y. Fushiwaki, Y. Hagiwara, Shuichi Hamada, T. Hamamura, Y. Iwase, Y. Kajiwara, Y. Kasahara, Y. Kato, M. Kawabata, E. Kitada, K. Kaneko, Yuko Kizaki K. Kubo, D. Miura, K. Mashiko, F. Mizuhashi, D. Muramatsu, M. Nakajima, T. Nakamura, H. Oishi, Toshiaki Sasaki, S. Shimada, C. Takahashi, Y. Takeda, S. Wakuri, N. Yajima, S. Yajima, T. Yatsushiro, Application of the improved BALB/c 3T3 cell transformation assay to the examination of the initiating and promoting activities of chemicals: The second interlaboratory collaborative study by the non-genotoxic carcinogen study group of Japan, ATLA-Altern. Lab. Anim. 38 (2010) 11-27.

[18] S. Plöttner, H.U. Käfferlein, T.Brüning, Miniaturization of cytotoxicity tests for concentration range-finding studies prior to conducting the $\mathrm{pH} 6.7$ Syrian hamster embryo cell-transformation assay, Mutat. Res 755 (2013) 108-114.

[19] M.H. Wu, S.B. Huang, G.B. Lee, Microfluidic cell culture for drug research. Roy. Soc. Ch. 10 (2010) 939-956.

[20] Meyvantsson, D.J. Beebe, Cell culture models in microfluidic systems. Annu. Rev. Anal. Chem. 1 (2008) 423-449.

[21] L. Kim, Y.C Toh, J. Voldman, H. Yu, A practical guide to microfluidic culture of adherent mammalian cells, Roy. Soc. Ch 7 (2007) 681-694.

[22] A.L. Paguirigan, D.J. Beebe, From the cellular perspective: exploring differences in the cellular baseline in macrosclale and microfluidic cultures. Roy. Soc. Ch. 1 (2009) 182-195.

[23] K. Sasaki, S. Bonhenberger, K. Hayashi, T. Kunkelmann, D. Muramatsu, A. Poth, A. Sakai, S. Salovaara, N. Tanaka, B.C. Thomas, M. Umeda, Photo catalogue for the classification of foci in the Balb/c 3T3 cell transformation assay, Mutat. Res. 744 (2012a), pp. 42-53.

[24] K. Ohomori, In vitro assays for the prediction of Tumorigenic Potential of nongenotoxic carcinogens, J Health Sci. 55(1) (2009) 20-30.

[25] S. Hoffmann, L.A. Hothorn, L. Edler, A. Kleensang, M. Suzuki, P. Phrakonkham, D. Gerhard, Two new approaches to improve the analysis of BALB/c 3T3 cell transformation assay data, Mutat. Res., 744 (2012) 36-41.

[26] D. Hanahan, R.A. Weinberg, Hallmarks of cancer: The next generation, Cell 144 (2011) 646-674.

[27] D. Hanahan, L.M. Coussens, Accessories to a crime: functions of cells recruited to the tumor microenvironment, Cancer Cell 21 (2012) 309-322.

[28] R.J. DeBerardinis, C.B. Thompson, Cellular metabolism and disease: What do metabolic outliers teach us?, Cell 148 (2012) 1132-1140 